\title{
Increased soluble and membrane-bound PD-L1 contributes to immune regulation and disease progression in patients with tuberculous pleural effusion
}

\author{
XUE PAN $^{1 *}$, ANYUAN ZHONG $^{1 *}$, YUFEI XING $^{1}$, MINHUA SHI $^{1}$, BIN QIAN $^{1}$, \\ TONG ZHOU $^{1}$, YONGJING $\mathrm{CHEN}^{2,3}$ and XUEGUANG ZHANG ${ }^{2,3}$ \\ ${ }^{1}$ Department of Respiration, The Second Affiliated Hospital of Soochow University, Suzhou, Jiangsu 215004; \\ ${ }^{2}$ Department of Immunology, Medical College of Soochow University, Suzhou, Jiangsu 215006; \\ ${ }^{3}$ Key Laboratory of Infection and Immunity, Soochow University, Suzhou, Jiangsu 200241, P.R. China
}

Received April 2, 2015; Accepted April 22, 2016

DOI: $10.3892 / \mathrm{etm} .2016 .3611$

\begin{abstract}
Soluble and membrane-bound programmed death ligand-1 (sPD-L1 and mPD-L1, respectively) have been demonstrated to participate in the immune suppression of non-small cell lung cancer. However, the contribution of sPD-L1 and mPD-L1 to immune regulation and disease progression in patients with pleural effusions remains unknown. The present study evaluated the levels of sPD-L1 and membrane-bound PD-1/PD-L1 in the peripheral blood and pleural effusions of patients with tuberculous pleural effusion (TPE), malignant pleural effusion (MPE) and non-tuberculous non-malignant pleural effusion (n-TB n-M). Furthermore, selected $\mathrm{T}$ lymphocytes and cluster of differentiation (CD) $14^{+}$monocytes were co-cultured to investigate the potential effect of the PD-1/PD-L1 pathway in TPE. Levels of sPD-L1 and PD-L1 on CD14 ${ }^{+}$monocytes were increased in the TPE group, as compared with the MPE and n-TB n-M groups. Furthermore, sPD-L1 levels and the expression levels of PD-L1 on $\mathrm{CD}_{14}{ }^{+}$monocytes were demonstrated to be positively correlated with interferon (IFN) $\gamma$ concentration in pleural effusions. Therefore, IFN- $\gamma$ may increase the expression of PD-L1 on $\mathrm{CD}_{1}{ }^{+}$monocytes in vitro. Cell counting kit-8 analysis demonstrated that anti-PD-L1 antibody was able
\end{abstract}

Correspondence to: Professor Minhua Shi, Department of Respiration, The Second Affiliated Hospital of Soochow University, 1055 Sanxiang Road, Suzhou, Jiangsu 215004, P.R. China

E-mail: shiminhua@163.com

Dr Yongjing Chen, Department of Immunology, Medical College of Soochow University, 1055 Sanxiang Road, Suzhou, Jiangsu 215006, P.R. China

E-mail: wellchen_cn@sina.com

*Contributed equally

Key words: tuberculous pleural effusion, soluble programmed death ligand-1, programmed death ligand-1, antibody, interferon- $\gamma$ to partially reverse the proliferation of $\mathrm{T}$ lymphocytes in the co-culture system. The results of the present study indicated that SPD-L1 or mPD-L1 are associated with the immune regulation and disease progression of TPE, and may serve as possible biomarkers of TPE. Furthermore, sPD-L1 and the PD-1/PD-L1 pathway of TPE may be associated with the Th1 immune response; therefore, an anti-PD-1/PD-L1 pathway suggests a potential immune therapy strategy for the treatment of TPE.

\section{Introduction}

Tuberculosis (TB) remains one of the top three deadly diseases worldwide with 1.4 million deaths reported in 2011 (1). TB pleurisy is the most common type of extra-pulmonary tuberculosis, accounting for $10-20 \%$ of patients with tuberculous and $10-30 \%$ of patients with TB pleurisy suffer from pleural effusions (PFs) (2). TB-associated PF results from the infiltration of pleural space by $M$. tuberculosis $(M . t b)$ antigens or bacilli, which induce a strong Th1 immune response (3). Currently, non-invasive methods including conventional chest X-ray, chest computed tomography scan, ultrasonography, pleural fluid laboratory tests, thoracocentesis, pleural biopsy and thoracoscopy are used to determine the existence and etiology of PFs (4). Pleural fluid M.tb culture remains the gold standard criterion with a diagnostic detection rate of $<20 \%(5)$.

Programmed death ligand 1 (PD-L1), which is also known as CD274, has been well-characterized as a critical membrane-bound co-stimulatory molecule that inhibits immune responses through its receptor, programmed death 1 (PD-1) by downregulating $\mathrm{T}$ cell activation and cytokine secretion $(6,7)$. PD-L1 is constitutively expressed in various immunocytes, including T cells, B cells, dendritic cells (DCs), macrophages and a wide range of tumor cell lines and solid tumor tissues, and the expression of PD-L1 is further upregulated following activation (8). PD-1 is a type I glycoprotein and its expression can be induced in T cells, B cells, natural killer $\mathrm{T}$ cells and activated monocytes (9). Various co-stimulatory molecules express PD-1 in two forms, the soluble (sPD-L1) and membrane-bound (mPD-L1) forms. sPD-L1 has been 
Table I. Basic characteristics of the subjects enrolled in the present study.

\begin{tabular}{lccc}
\hline Characteristic & TPE & MPE & n-TB n-M \\
\hline Number of subjects & 24 & 30 & 14 \\
Age (years) & $46.42 \pm 23.44^{\mathrm{a}}$ & $67.70 \pm 8.19$ & $63.36 \pm 17.27$ \\
Gender (M/F) & $20 / 4$ & $13 / 17$ & $12 / 2$ \\
Pleural effusion side (right/left/bilateral) & $7 / 17 / 0$ & $16 / 12 / 2$ & $3 / 3 / 8$ \\
Presence of fever admission (yes/no) & $14 / 10$ & $2 / 28$ & $4 / 10$ \\
Smoking (yes/no) & $8 / 16$ & $7 / 23$ & $4 / 10$ \\
Ethnic group & Chinese & Chinese & Chinese \\
\hline
\end{tabular}

TPE, tuberculous pleural effusion; MPE, malignant pleural effusion; n-TB n-M, non-tuberculous and non-malignant pleural effusion. Data are presented as the mean \pm standard deviation. ${ }^{a} \mathrm{P}<0.0001$, vs. the MPE and n-TB n-M groups.

demonstrated in various members of the B7 superfamily, including OX40 and B7-H3 (10,11). sPD-L1 was initially identified in the blood serum of patients with non-small cell lung cancer, and increased SPD-L1 expression is associated with lung cancer TNM staging and the patient's clinical response to treatment (12). Increased levels of serum sPD-L1 have also been detected in other solid tumors, such as aggressive renal cell carcinoma, breast cancer and autoimmune diseases, including systemic lupus erythematosus, rheumatoid arthritis and type 2 diabetes mellitus (13-16). Therefore, sPD-L1 may have important roles in the regulation of co-stimulatory signals. Th1 cytokines, including interferon (IFN) $-\gamma$, tumor necrosis factor (TNF)- $\alpha$ and interleukin-2, have also been demonstrated to have critical roles in granuloma formation and the damage of tubercle bacilli within macrophages (17). However, whether sPD-L1 and mPD-L1 are involved in immune regulation and disease progression of TPE has yet to be elucidated. In the present study, the immune regulatory role of sPD-L1 and the PD-1/PD-L1 pathway involved in TPE, and the correlation with Th1 immune response, will be explored. In addition, the possibility of a potential immune-checkpoint in the treatment of TPE will be investigated.

\section{Materials and methods}

Subjects. The present study was approved by the Ethics Committee of The Second Affiliated Hospital of Soochow University (Suzhou, China). Written informed consents for the collection of blood and PF samples and subsequent analysis were obtained from all participants prior to the initiation of the study. Fresh PF and peripheral blood (PB) samples were harvested from 68 subjects. Of 68 patients with evaluable data, 24 were assigned to the tuberculous pleural effusion (TPE) group, 30 were assigned to the malignant pleural effusion (MPE) group and 14 were assigned to the non-tuberculous non-malignant pleural effusion (n-TB n-M) group. Within the $\mathrm{n}-\mathrm{TB} \mathrm{n}-\mathrm{M}$ group, the 14 participants presented with systemic lupus erythematosus (SLE; $n-1)$, heart failure $(n=3)$, pneumonia $(n=5)$ and an unknown medical condition $(n=5)$. Clinical and microbiological characteristics of subjects were obtained from the patient records and are reported in Table I.

Patients were diagnosed with TPE on the basis of: i) Presence of acid fast bacilli in a pleural fluid specimen, growth of $M . t b$ from pleural fluid, or demonstration of granulomatous pleurisy on closed pleural biopsy specimen in the absence of any evidence of other granulomatous diseases; ii) an exudative lymphocytic effusion with an adenosine deaminase level of $>40 \mathrm{U} / 1$, a positive purified protein derivative skin test result and the exclusion of any other potential causes of pleurisy; iii) or a good response to TB treatment (18). Exudative effusion was classified according the criteria outlined by Light et al (19). A diagnosis of MPE was established following demonstration of malignant cells in pleural fluid and/or pleural biopsy specimen (20). SLE was diagnosed according to the criteria outlined by the American College of Rheumatology in 1982 (21). Cardiogenic PF was diagnosed according to the symptoms and laboratory tests of heart failure. Para-pneumonic PF was diagnosed following evidence of pulmonary infections associated with acute febrile illness, pulmonary infiltrates, purulent sputum and response to antibiotic treatment (22). Ten PB samples were collected from healthy clinical trial volunteers. At the time of sample collection, none of the patients had received anti-TB therapy, anticancer therapy, corticosteroids, other non-steroid drugs or invasive procedures directed into the pleural cavity.

Determination of immune cell profiles in PF and PB. PF monocyte cells (PEMCs) were obtained by Ficoll-Hypaque density centrifugation at $543 \times \mathrm{g}$ for $30 \mathrm{~min}$. PEMCs $\left(\sim 5 \times 10^{5}\right.$ cells/test) and PB (50 $\mu \mathrm{l} /$ test) were surface-stained with fluorescein isothiocyanate (FITC)-labeled anti-human CD4 (clone SK3) and CD8 (clone OKT8) monoclonal antibodies (mAbs) and (eBioscience, Inc., San Diego, CA, USA), FITC-labeled anti-human CD14 mAb (clone 61D3; eBioscience, Inc.), phycoerythrin-labeled anti-human PD-1 (clone EH12. 2H7) and PD-L1 (clone 29E. 2A3) mAbs (BioLegend, Inc., San Diego, CA, USA) for $30 \mathrm{~min}$ at $4^{\circ} \mathrm{C}$ according to the manufacturer's protocol. A blank was used for the control. For PEMCs, $500 \mu \mathrm{l}$ fluorescence-activated cell sorting (FACS) buffer (Beckman Coulter, Inc., Brea, CA, USA) was used for flow cytometry acquisition. For the blood samples, $300 \mu 1$ erythrocyte lysis buffer (Beckman Coulter, Inc.) was added to the cell suspensions following antibody staining and incubated for a further $10 \mathrm{~min}$ at $42^{\circ} \mathrm{C}$, followed by the addition of $500 \mu \mathrm{l} \mathrm{FACS}$ buffer for flow cytometry acquisition. Data were acquired on a 2-color flow cytometer (Beckman Coulter cytomics FC500) 
and analyzed with Expo32 MultiCOMP software version 1.2 (both Beckman Coulter, Inc.).

Determination of $S P D-L 1$ and IFN- $\gamma$ levels. PF $(50 \mathrm{ml})$ and PB $(5 \mathrm{ml})$ samples were centrifuged at $543 \mathrm{x}$ g for $10 \mathrm{~min}$ and the supernatants were stored at $-80^{\circ} \mathrm{C}$ for subsequent ELISA assays. Expression levels of sPD-L1 in PFs and sera were determined in duplicate wells using the SPD-L1 ELISA system, as previously described by our group (9). Expression levels of IFN- $\gamma$ were analyzed using a commercial ELISA kit (cat no. EK0373; Boster Biological Technology, Ltd., Wuhan, China) according to the manufacturer's protocol.

Determination of PD-L1 on $\mathrm{CD} 14^{+}$monocytes. PB monocyte cells (PBMCs) were collected from healthy donors by Ficoll-Hypaque density centrifugation and seeded into 6-well plate at a density of $3 \times 10^{5}$ cells/well. TPE were co-cultured with PBMCs for $48 \mathrm{~h}$ at $37^{\circ} \mathrm{C}$ in an incubated atmosphere containing $5 \% \mathrm{CO}_{2}$. Alterations in the levels of PD-L1 on $\mathrm{CD} 14^{+}$monocytes were evaluated by flow cytometry.

Determination of cell proliferation. PEMCs from TPE were collected and $\mathrm{CD}^{+} 4^{+}$monocytes were isolated using human CD14 MicroBeads (Miltenyi Biotec, GmbH, Bergisch Gladbach, Germany) with positive selection. Subsequently, purified $\mathrm{T}$ cells were obtained using a $\mathrm{T}$ cell negative kit (Stemcell Technologies, Inc., Vancouver, BC, Canada). CD14 ${ }^{+}$ monocytes and $\mathrm{T}$ lymphocytes were seeded at a ratio of 1:5 into a 96-well plate pre-coated with functional anti-human CD3 mAb (2 $\mu \mathrm{g} / \mathrm{ml}$; cat no. IM1304; Immunotech S.A.S, Marseille, France). Anti-PD-L1 mAb (2H11; $1 \mu \mathrm{g} / \mathrm{ml})$, which was developed by our own group (9), or the control antibody mouse anti-human IgG (cat no. 6602872) was added into the medium. Following co-culturing for 3-5 days, cell proliferation was detected by a cell counting kit-8 (CCK-8; cat no. CK04).

Statistical analysis. Data were analyzed using GraphPad Prism 5.0 (Graph Pad Software Inc., San Diego, CA, USA) and SPSS 17.0 (IBM, Chicago, USA). Data with normal distribution were analyzed with paired and unpaired t-test, and one-way analysis of variance with Newman-Keuls test. Data without a normal distribution were analyzed with Wilcoxon matched pairs test, Mann-Whitney U-test and Kruskal-Wallis test with Dunn's Multiple Comparison. Spearman correlation analysis was used to calculate the correlation coefficient. $\mathrm{P}<0.05$ was considered to indicate a statistically significant difference.

\section{Results}

Immune cell profiles in PF differ from those in PB. Immune cell profiles of $\mathrm{CD}^{+}{ }^{+}$and $\mathrm{CD} 8^{+}$cells, which included the expression of PD- 1 on $\mathrm{CD} 4^{+}$cells and $\mathrm{CD} 8^{+}$cells, and $\mathrm{CD} 14^{+}$ monocytes, which included the expression of PD-L1 on CD14 ${ }^{+}$ monocytes in $\mathrm{PF}$ and in $\mathrm{PB}$ from 41 subjects, were analyzed (Tables II and III). With the exception of $\mathrm{CD} 8^{+}$cells (which were lower in $\mathrm{PF}$ compared with those in $\mathrm{PB}$ ), expressions of PD- 1 on $\mathrm{CD}^{+}$cells, $\mathrm{PD}-1$ on $\mathrm{CD} 8^{+}$cells, $\mathrm{CD} 14^{+}$monocytes and $\mathrm{PD}-\mathrm{L} 1$ on $\mathrm{CD} 14^{+}$monocytes in $\mathrm{PF}$ were higher compared with those in $\mathrm{PB}$, and the $\mathrm{p}$-values were $\mathrm{P}<0.0001, \mathrm{P}<0.0001$, $\mathrm{P}=0.0003$ and $\mathrm{P}=0.0123$, respectively.. The ratio of $\mathrm{CD} 4^{+} / \mathrm{CD}^{+}$ 
Table III. Comparison of immune subsets in pleural effusion and peripheral blood.

\begin{tabular}{|c|c|c|c|c|c|c|}
\hline & PF vs. PB & TB (PF vs. PB) & $\begin{array}{l}\text { TPE vs. MPE } \\
\text { vs. n-TB n-M }\end{array}$ & & & \\
\hline Subset (\%) & P-value & P-value & P-value & TPE $(n=18)$ & Non-TPE $(n=23)$ & P-value \\
\hline $\mathrm{CD}^{+}{ }^{+}$ & 0.0919 & 0.0007 & 0.0183 & $49.8(41.6-61.2)$ & $44.3(22.8-55.9)$ & 0.0902 \\
\hline $\mathrm{CD}^{+}+$ & $<0.0001$ & $<0.0001$ & 0.0001 & $18.7(13.5-23.4)$ & $8.2(3.9-14.3)$ & $<0.0001$ \\
\hline $\mathrm{CD}^{+} / \mathrm{CD}^{+}$ratio & $<0.0001$ & $<0.0001$ & 0.0054 & $2.5(2.1-5.0)$ & $3.1(2.6-8.8)$ & 0.0853 \\
\hline $\mathrm{CD} 14^{+}$ & 0.0003 & 0.2066 & 0.0434 & $30.5(6.9-63.0)$ & $62.4(34.9-81.8)$ & 0.0168 \\
\hline $\mathrm{CD}^{+}{ }^{+} \mathrm{PD}-1^{+}$ & $<0.0001$ & 0.0035 & 0.3330 & $38.6(24.6-44.8)$ & $47.7(24.9-57.8)$ & 0.1619 \\
\hline $\mathrm{CD} 8^{+} \mathrm{PD}^{-1} 1^{+}$ & $<0.0001$ & 0.0131 & 0.0571 & $19.3(10.7-25.9)$ & $34.7(14.8-43.0)$ & 0.0195 \\
\hline $\mathrm{CD}^{+} / \mathrm{CD}^{+}{ }^{+} \mathrm{PD}-1^{+}$ratio & 0.7558 & 0.1914 & 0.1494 & $2.3(1.21-2.45)$ & $1.4(1.17-1.90)$ & 0.0719 \\
\hline $\mathrm{CD}_{14}{ }^{+} \mathrm{PD}-\mathrm{L} 1^{+}$ & 0.0123 & $<0.0001$ & $<0.0001$ & $92.0(76.9-97.3)$ & $21.2(11.3-40.0)$ & $<0.0001$ \\
\hline
\end{tabular}

Data are presented as the median (interquartile range) from subjects with TPE $(n=18)$ and non-TPE $(n=23)$. PF vs. PB, the difference between immune subsets for the same subject; TB (PF vs. PB), the difference between immune subsets for the same subject diagnosed with TB pleurisy; TPE vs. MPE vs. n-TB n-M, the difference between immune subsets in different pleural effusions. Statistically significant $(\mathrm{P}<0.05)$ differences are indicated in bold. PF, pleural effusion; PB, peripheral blood; TPE, tuberculous pleural effusion; MPE, malignant pleural effusion; n-TB $\mathrm{n}-\mathrm{M}$, non-tuberculous and non-malignant pleural effusion; $\mathrm{CD}$, cluster of differentiation; PD, programmed death.

cells was significantly increased in $\mathrm{PF}$, as compared with $\mathrm{PB}$ $(\mathrm{P}<0.001$; Tables II and III). For subjects who were diagnosed with TPE, with the exception of $\mathrm{CD} 14^{+}$monocytes $(\mathrm{P}=0.2066)$, expression levels of $\mathrm{CD} 4^{+}, \mathrm{CD} 8^{+}, \mathrm{CD} 4^{+} \mathrm{PD}-1^{+}$and $\mathrm{CD} 8^{+} \mathrm{PD}-1^{+}$ cells and $\mathrm{CD} 14^{+} \mathrm{PD}-\mathrm{L}^{+}$monocytes in $\mathrm{PF}$ were significantly different from those in $\mathrm{PB}$. In addition, in subjects who were diagnosed with TPE, expression of CD $4^{+} \mathrm{PD}-1$ and $\mathrm{CD} 8^{+} \mathrm{PD}-1^{+}$ cells were higher in $\mathrm{PF}$ than $\mathrm{PB}$, and $\mathrm{P}$-values were $\mathrm{P}=0.0035$ and $\mathrm{P}=0.0131$. Percentage of $\mathrm{CD}^{+}$cells was increased in $\mathrm{PF}$, as compared with $\mathrm{PB}$, whereas the opposite was true for $\mathrm{CD}^{+}$ cells. Furthermore, the expression levels of PD-L1 on CD14 ${ }^{+}$ monocytes in PF were significantly increased, as compared with those in PB.

For immune subsets in $\mathrm{PB}$, the percentage of $\mathrm{CD} 4^{+}$cells and the level of PD-L1 on $\mathrm{CD} 14^{+}$monocytes in the MPE group were significantly increased compared with those in the TPE and $\mathrm{n}-\mathrm{TB}$ n-M groups $(\mathrm{P}=0.018$ and $\mathrm{P}=0.0128$, respectively). No significant differences in $\mathrm{PB}$ were detected in the other subsets (data not shown). For immune subsets in PF, the percentage of $\mathrm{CD}^{+}$cells was increased in the TPE group, as compared with the MPE and the n-TB n-M groups. Furthermore, increased expression of PD-L1 on $\mathrm{CD} 14^{+}$monocytes was detected in the TPE group, as compared with the MPE and n-TB n-M groups (Fig. 1; Tables II and III). Expression of PD-L1 was increased in the TPE group, as compared with the MPE and n-TB n-M groups at the mRNA level.

Subjects diagnosed with TPE and non-TPE were analyzed for potential biomarkers that may distinguish between them. No differences in the total expression levels of $\mathrm{CD}^{+}, \mathrm{CD}^{+}$, CD $4{ }^{+} \mathrm{PD}-1^{+}$and $\mathrm{CD} 8{ }^{+} \mathrm{PD}-1^{+}$cells, and $\mathrm{CD} 14^{+}$and $\mathrm{CD} 14^{+} \mathrm{PD}-\mathrm{L} 1^{+}$ monocytes were detected in PB (data not shown). For immune subsets in $\mathrm{PF}$, an increased level $\mathrm{CD} 14^{+} \mathrm{PD}-\mathrm{L}^{+}$monocytes was demonstrated in the TPE group, as compared with the non-TPE group (Table III). The P-values for CD14 ${ }^{+} \mathrm{PL}-\mathrm{L1}^{+}$and $\mathrm{CD} 8{ }^{+}$cells and $\mathrm{CD} 8{ }^{+} \mathrm{PD}-1^{+}$cells were $\mathrm{P}<0.0001, \mathrm{P}<0.0001$ and $\mathrm{P}=0.0195$, respectively. Consistent with the result of $\mathrm{CD}^{+}$ cells above, the levels of $\mathrm{CD} 8^{+}$cells were increased in the TPE group, as compared with the MPE and the n-TB n-M groups; whereas the expression of PD- 1 on $\mathrm{CD}^{+}$cells was decreased in TPE. Thus, sPD-L1 and mPD-L1 are involved in the immune regulation and disease progression of TPE.

Expression levels of $S P D-L 1$ and IFN- $\gamma$ are elevated in $T P E$. Expression levels of sPD-L1 were increased in PF, as compared with $\mathrm{PB}$ for the same subject $(\mathrm{P}=0.0033$; Fig. 2A and B). Furthermore, increased expression levels of sPD-L1 were detected in the TPE group, as compared with the MPE and n-TB n-M groups (both $\mathrm{P}<0.05$; Fig. 2C); however, no significant differences were detected between the MPE and n-TB n-M PF groups. Expression levels of sPD-L1 were increased in the TPE group, as compared with the non-TPE group (Fig. 2D; Table IV). Increased expression levels of IFN- $\gamma$ were detected in the TPE group, as compared with the MPE and n-TB n-M groups $(\mathrm{P}<0.05$; Fig. $2 \mathrm{E})$; however, no differences were detected between the MPE and n-TB n-M groups. Expression levels of IFN- $\gamma$ in the TPE group were significantly increased, as compared with the non-TPE group $(\mathrm{P}<0.0001$; Fig. 2F; Table IV). According to receiver operating curve analysis, levels of $>2.41 \mathrm{ng} / \mathrm{ml}$ sPD-L1 in PF were able to discriminate between the TPE and non-TPE groups, with a specificity ( $\mathrm{Sp}$ ) of $82.9 \%$, sensitivity $(\mathrm{Se})$ of $82.6 \%$ and area under the curve (AUC) of 0.840 . Using an IFN $-\gamma$ expression level of $>31.06 \mathrm{pg} / \mathrm{ml}$ as the cut off value, the TPE group was successfully differentiated from the non-TPE group (Sp, 95.1\%; Se, 87\%; AUC, 0.923). Using a value of $>72.75 \% \mathrm{PD}-\mathrm{L} 1$ on $\mathrm{CD}_{14}{ }^{+}$monocytes as the cut off value, the TPE group was successfully differentiated from the non-TPE group (Sp, 92.7\%; Se, 78.3\%; AUC, 0.898) (Table IV). Furthermore, a combination of sPD-L1, IFN- $\gamma$ and PD-L1 on CD14 ${ }^{+}$monocytes demonstrated an increased AUC of 0.962 (Sp, 100\%; Se, 91.3\%), as compared with the parameters alone, using multivariate analysis. SPD-L1 and PD-L1 on 
Table IV. Se and Sp of pleural effusion indices in distinguishing TPE from non-TPE.

\begin{tabular}{|c|c|c|c|c|c|c|c|}
\hline Parameter & TPE & Non-TPE & P-value & $\mathrm{Se}(\%)$ & $\mathrm{Sp}(\%)$ & AUC & Cutoff \\
\hline sPD-L1 & $4.2(2.53-6.3)$ & $1.7(0.90-2.26)$ & $<0.0001$ & 82.6 & 82.9 & 0.840 & $2.41 \mathrm{ng} / \mathrm{ml}$ \\
\hline IFN- $\gamma$ & $338.6(128.7-626.9)$ & $9.4(6.5-18.6)$ & $<0.0001$ & 87.0 & 95.1 & 0.923 & $31.06 \mathrm{pg} / \mathrm{ml}$ \\
\hline $\mathrm{CD}_{14}{ }^{+} \mathrm{PD}-\mathrm{L}^{+}$ & $89.9(73.5-97.9)$ & $16.9(12.6-44.8)$ & $<0.0001$ & 78.3 & 92.7 & 0.898 & $72.75 \%$ \\
\hline $\mathrm{CD}_{1} 4^{+} \mathrm{sPD}_{\mathrm{L}} 1^{+}{ }^{+} \mathrm{FN}-\gamma^{+} \mathrm{PD}-\mathrm{L} 1^{+}$ & None & None & None & 91.3 & 100.0 & 0.962 & None \\
\hline
\end{tabular}

Data are presented as the median (interquartile range) from subjects with TPE $(n=24)$ and non-TPE $(n=44)$. Statistically significant $(\mathrm{P}<0.05)$ differences are indicated in bold. Se, sensitivity; Sp, specificity; TPE, tuberculous pleural effusion; AUC, area under the curve; sPD-L1, soluble programmed death ligand-1; IFN, interferon.
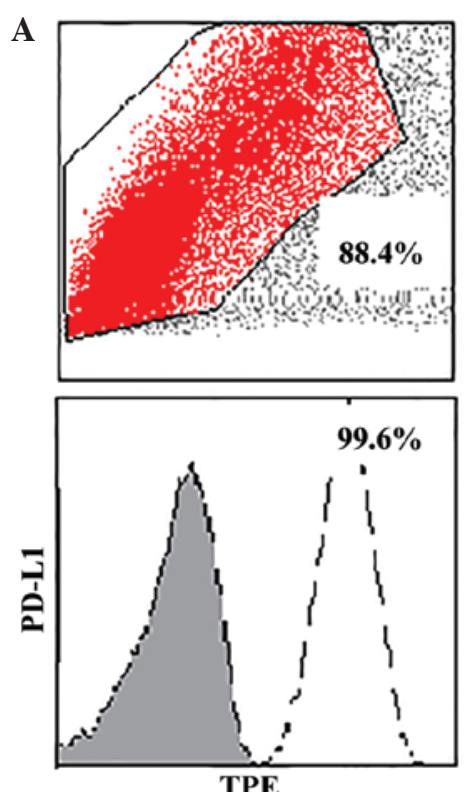
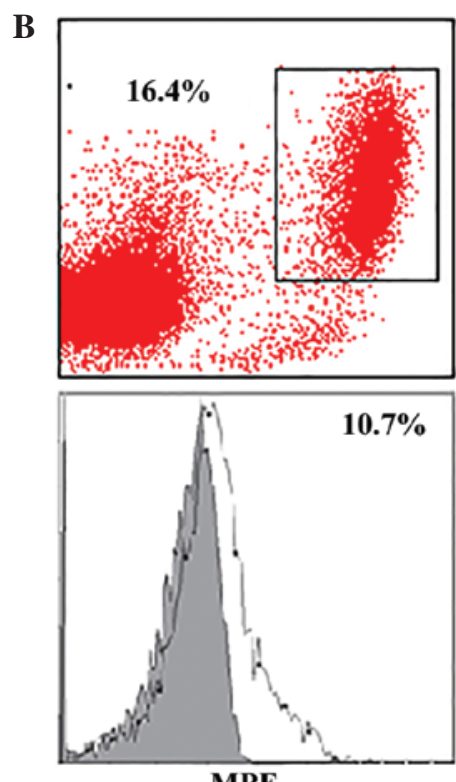

MPE

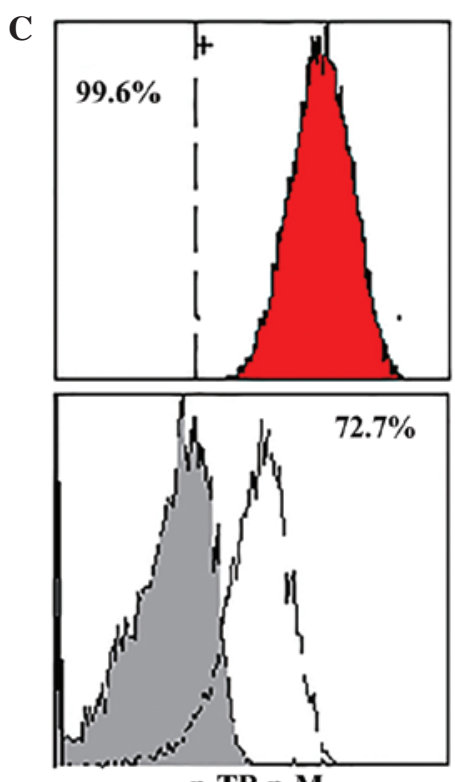

n-TB n-M

Figure 1. Expression of PD-L1 on CD14+ monocytes, as detected by flow cytometry. (A) Total distribution of pleural effusion monocyte cells from the subject who was diagnosed with tuberculous pleural effusion. (B) Expression of CD14 ${ }^{+}$monocytes in the proportion of region marked in (A). (C) Expression of PD-L1 on $\mathrm{CD}_{1} 4^{+}$monocytes in the region marked in (B). TPE, MPE and n-TB n-M demonstrate the expression of PD-L1 on CD14 ${ }^{+}$monocytes in different pleural effusions by flow cytometry. PD-L1, programmed death ligand-1; CD, cluster of differentiation; TPE, tuberculous pleural effusion; MPE, malignant pleural effusion; n-TB n-M, non-TB non-M pleural effusion.

$\mathrm{CD} 14^{+}$monocytes are higher in TPE, which suggests that they may serve as possible biomarkers of TPE.

IFN- $\gamma$ increases the expression levels of $S P D-L 1$ and $P D-L 1$ on $C D 14^{+}$monocytes in PBMCs. The results of flow cytometry demonstrated that the expression levels of PD-L1 on CD14 ${ }^{+}$ monocytes were improved in the TPE-stimulated group, as compared with the control $(\mathrm{P}<0.05$; Fig. 3A). In order to investigate the possible correlation between IFN $-\gamma$ and mPD-L1 in PF, all the 68 subjects were studied. The results of this analysis demonstrated that the expression of PD-L1 on $\mathrm{CD}_{1} 4^{+}$monocytes was significantly positively correlated with the level of IFN- $\gamma$, based on Spearman's correlation $(\mathrm{P}<0.0001$; Fig. 3B); however, no significant differences were detected in the TPE group ( $r=0.16 ; \mathrm{P}=0.4552)$. In addition, Spearman's correlation analysis of sPD-L1 demonstrated that SPD-L1 expression levels were positively correlated with IFN- $\gamma,(\mathrm{P}=0.0004$; Fig. $3 \mathrm{C})$ and PD-L1 on CD14 ${ }^{+}$monocytes $(r=0.2984 ; \mathrm{P}=0.0166)$. Thus, he immune mechanism of
SPD-L1, and the PD-1/PD-L1 pathway involved in TPE are associated with Th1 immune response.

Anti-PD-L1 mAb enhances the proliferation of T lymphocytes in co-cultivation with $\mathrm{CD} 4^{+}$monocytes. To determine the effect of PD-L1 in TPE, enriched T cells and purified CD14 ${ }^{+}$ monocytes were co-cultured and the proliferation of $\mathrm{T}$ cells in the co-culture system was evaluated by CCK- 8 kit. The results demonstrated that $\mathrm{T}$ cells were activated in association with anti-CD3 mAb following 4-day stimulation $(\mathrm{P}<0.0001$; Fig. 3D). Notably, supplementing the medium with PD-L1 antibody (2H11) partially reversed this effect ( $\mathrm{P}=0.005$; Fig. 3D). Anti-PD-1/PD-L1 pathway is a potential immune-checkpoint in the treatment of TPE.

\section{Discussion}

Tuberculous PF is usually the result of a hypersensitivity reaction to TB protein with chronic inflammatory infiltration by 
A

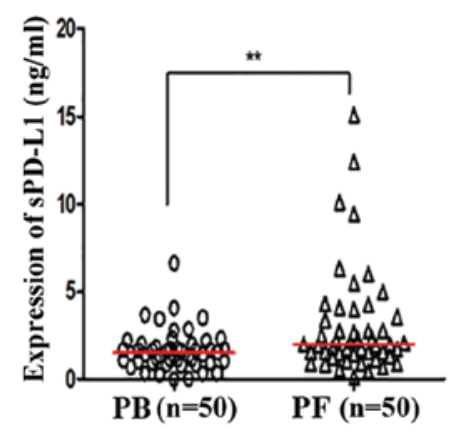

D

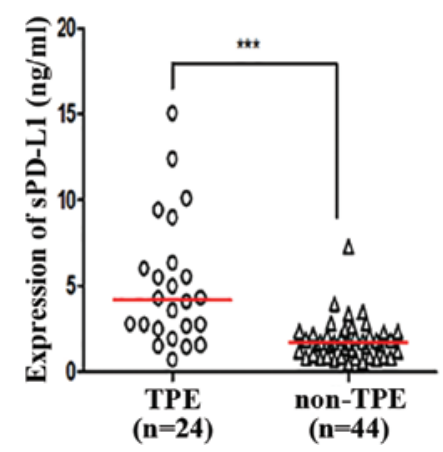

B

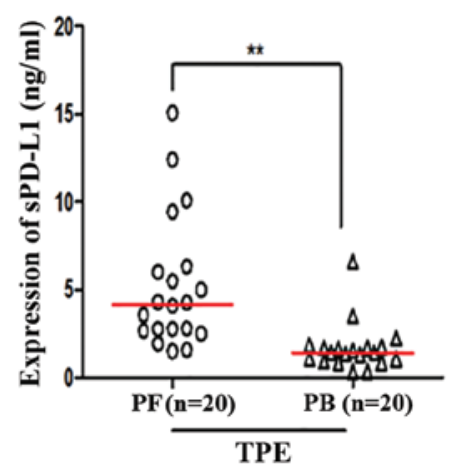

$\mathbf{E}$

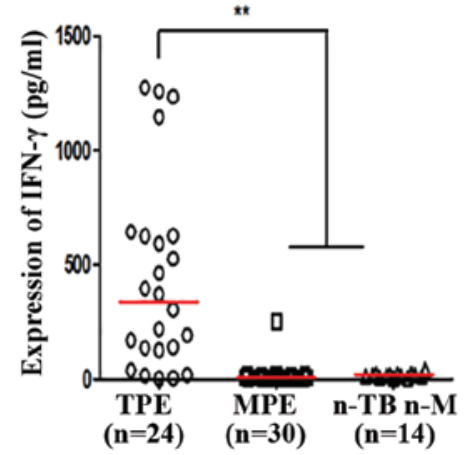

C

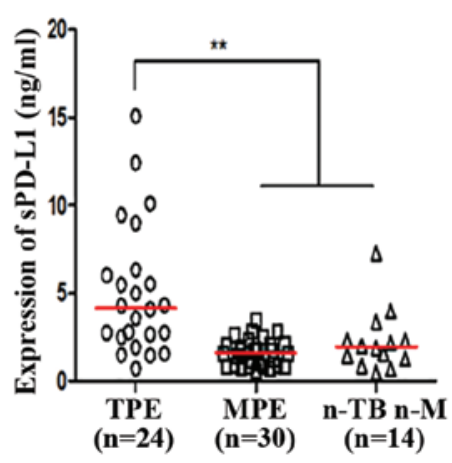

$\mathbf{F}$

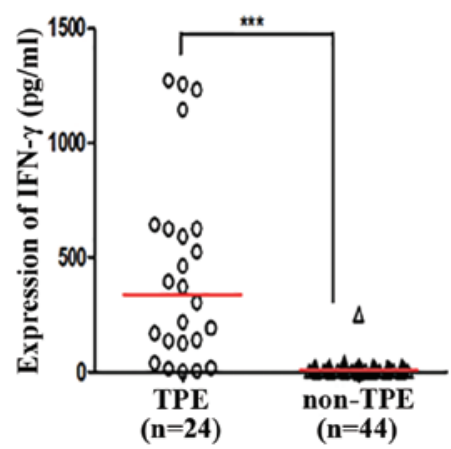

Figure 2. (A) Expression of sPD-L1 in PB and PF. (B) Expression of sPD-L1 in PF and PB in patients diagnosed with TPE. (C) Expression of sPD-L1 in the TPE, MPE and n-TB n-M groups. (D) Expression of sPD-L1 in patients diagnosed with TPE and non-TPE. (E) Expression of IFN- $\gamma$ in the TPE, MPE and n-TB n-M groups. (F) Expression of IFN- $\gamma$ in patients diagnosed with TPE and non-TPE. ${ }^{* *} \mathrm{P}<0.05 ;{ }^{* * *} \mathrm{P}<0.0001$. sPD-L1, sPD-L1, soluble programmed death ligand-1; PF, pleural effusion; PB, peripheral blood; TPE, tuberculous pleural effusion; MPE, malignant pleural effusion; n-TB n-M, non-TB non-M pleural effusion; IFN, interferon.

immune cells, including monocytes/macrophages and natural killer cells, in the pleural cavity. Previous studies have demonstrated that the PD-1/PD-L1 pathway has an important role in the immune response during infection with $M . t b(23-25)$. However, the immune mechanism of the PD-1/PD-L1 pathway's involvement in TB is yet to be fully elucidated. The present study analyzed the expression levels of $\mathrm{mPD}-\mathrm{L} 1$ and sPD-L1 in the PB and PF in patients with PFs, since sPD-L1 acts as an essential cytokine in lung cancer (12). As hypothesized, the expression levels of PD-L1 on $\mathrm{CD} 14^{+}$monocytes and sPD-L1 were increased in the TPE group and the Th1 cytokine IFN- $\gamma$ in PF may enhance this effect. It was also determined that anti-PD-L1 mAb is able to partially reverse the attenuated proliferation of $\mathrm{T}$ lymphocytes mediated by high levels of PD-L1 binding to PD-1 in vitro.

An accumulation of lymphocytes, particularly $\mathrm{CD} 4^{+}$ $\mathrm{T}$ cells, in TPE has been well documented in previous studies, demonstrating that patients who were infected with $M$. $t b$ may exhibit a strong Th1 immune response to kill $M$. tb, particularly in the young (26-28). Therefore, the PD-1/PD-L1 pathway and increased SPD-L1 levels may deliver a potent negative co-inhibitory signal to T cells. Alternatively, this phenomenon may prevent excessive immune injury and maintain balance in the immune system (29). However, the underlying pathogenesis remains unclear. Amarnath et al (30) have previously demonstrated that human regulatory $\mathrm{T}$ (Treg) cells promote immune suppression through dendritic cell modulation via the upregulation of PD-L1. Trinath et al (31) have also reported that M. $t b$ may promote the expansion of Treg cells via the induction of
PD-L1 on dendritic cells. In the present study, high expression levels of membranous and soluble PD-L1 in TPE indicated that PD-1/PD-L1 and SPD-L1 may have a role in $M . t b$ infection via Treg cells.

Although both myeloid and T lymphocytes exhibit mPD-L1 expression, the release of SPD-L1 is a feature of the myeloid population $(32,33)$. The results of the present study demonstrated that the expression levels of sPD-L1 in PF were increased, as compared with PB; this may be due to the high concentrations of cytokines in the local pleural cavity. High concentration of IFN- $\gamma$ were consistently detected in TPE, supporting the hypothesis that IFN- $\gamma$ may serve as a diagnostic biomarker (34). Lee et al (35) have previously suggested that IFN- $\gamma$ may upregulate the expression of PD-L1 via IFN regulatory factor-1. The results of the present study also indicated that SPD-L1 may be released with matrix metalloproteinases from the surface of immune and tumor cells (9). Notably, the expression levels of PD-L1 on $\mathrm{CD}_{1} 4^{+}$monocytes increased when the PBMCs were supplemented with TPE; however, the proliferation rate remained lower than is achieved with commercial IFN- $\gamma$ stimulation (16). It is possible that IFN- $\gamma$ is not the only cytokine with a role in the TB microenvironment, other cytokines, such as IFN- $\alpha$, may also have an effect $(26,36)$. In a previous study it was reported that IFN- $\alpha$ and IFN- $\beta$ strongly inhibited IFN- $\gamma$ responsiveness and the production of type I cytokines (15); however, no differences in IFN- $\alpha$ levels were detected in the TPE, MPE and n-TB n-M groups in the present study.

Previous studies have determined high levels of IFN- $\gamma$ in PF with a cutoff point varying from 60 to $240 \mathrm{pg} / \mathrm{ml}$ for TPE (37-41). 
A

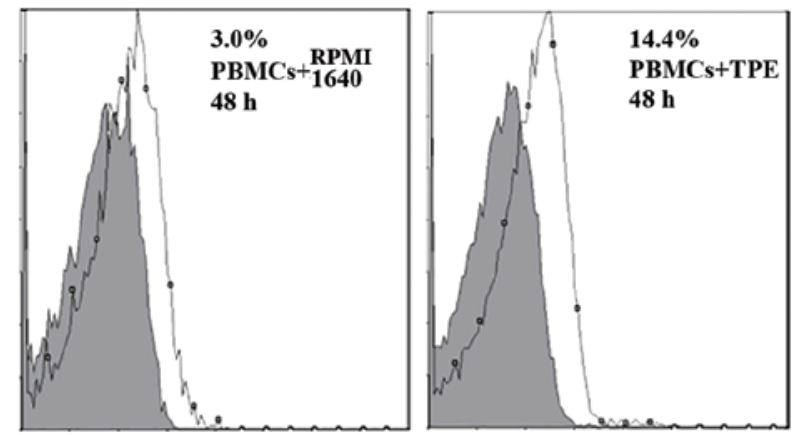

Expression of PD-L1 on CD14 monocytes

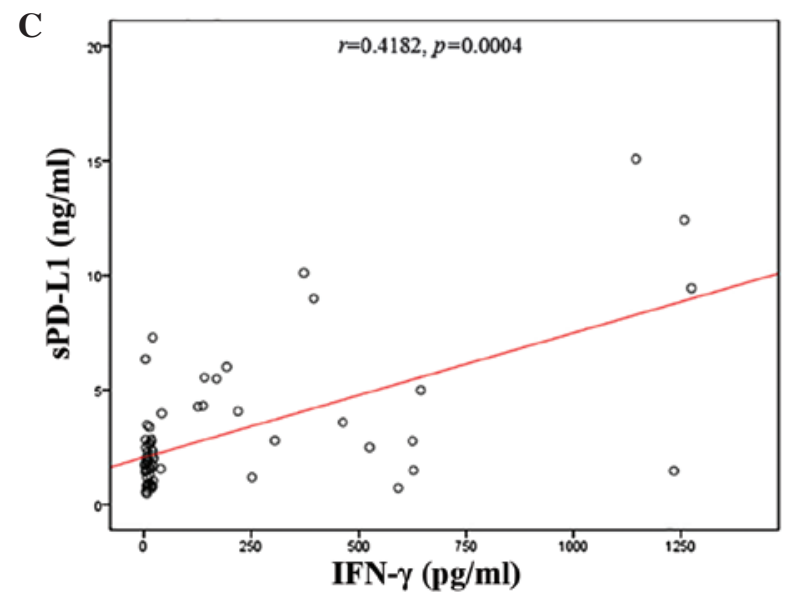

B

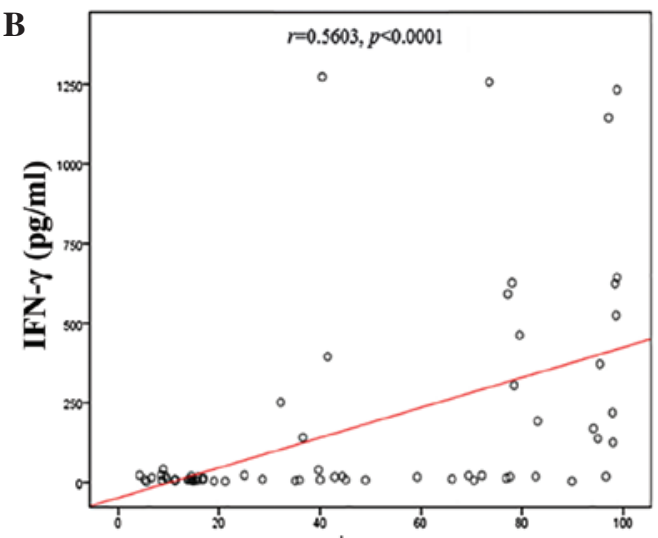

D CD14 + -PD-L1 (\%)

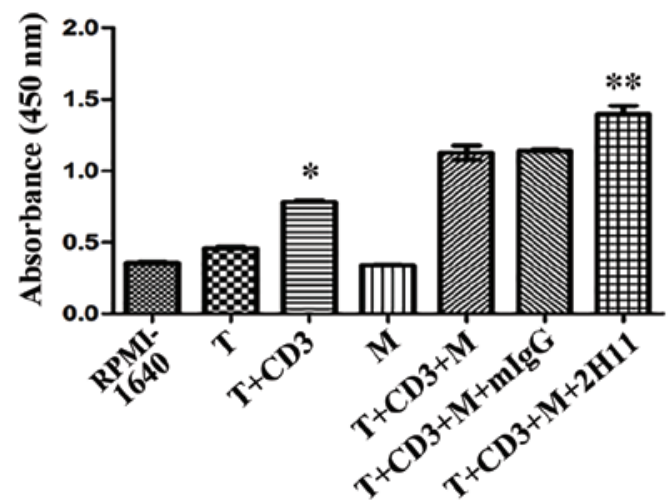

Figure 3. (A) PBMCs were supplemented with TPE and co-cultured for $48 \mathrm{~h}$, and the expression levels of PD-L1 on CD14 ${ }^{+}$monocytes were subsequently analyzed by flow cytometry. (B) A significant correlation was detected between IFN- $\gamma$ and PD-L1 on CD14 $4^{+}$monocytes in pleural effusion (P<0.0001) and (C) sPD-L1 and IFN- $\gamma$ in pleural effusion ( $\mathrm{P}=0.0004)$, respectively. (D) Effect of anti-PD-L1 monoclonal antibodies on the proliferation of T cells following 96-h co-cultivation with $\mathrm{CD} 14^{+}$monocytes was evaluated with a cell counting kit-8. ${ }^{*} \mathrm{P}<0.0001$, as compared with the $\mathrm{T}$ group; ${ }^{* *} \mathrm{P}=0.005$, as compared with the $\mathrm{T}+\mathrm{CD} 3+\mathrm{M}$ group. PBMCs, peripheral blood monocyte cells; TPE, tuberculous pleural effusion; PD-L1, programmed death ligand -1; CD, cluster of differentiation; sPD-L1, soluble PD-L1; IFN, interferon; T, T cells; CD3, agonistic CD3 antibody; M, monocytes that were separated from TPE; mIgG, mouse anti-human immunoglobulin G control antibody; 2H11, mouse anti-human PD-L1 antibody.

The result of the present study demonstrated a cutoff point of $31.06 \mathrm{pg} / \mathrm{ml}$ for IFN- $\gamma$. This difference may be attributed to the different methods, sample number and ethnicity of participants in the studies. Furthermore, in the present study it was demonstrated that anti-PD-L1 mAb enhanced the proliferation of $\mathrm{T}$ lymphocytes in co-cultivation with $\mathrm{CD} 14^{+}$monocytes, which indicated that an immune therapy method associated with PD-1/PD-L1 pathway in tuberculous PF may be developed.

In conclusion, the results of the present study suggested that sPD-L1 and mPD-L1 may be associated with the immune regulation and disease progression of TPE.SPD-L1. Increased levels of PD-L1 on $\mathrm{CD}_{1} 4^{+}$monocytes were detected in patients with TPE, indicating that PD-L1 may serve as a potential biomarker for TPE. The results also suggested that the immune mechanism of SPD-L1 and the PD-1/PD-L1 pathway in TPE are associated with the Th1 immune response; therefore, an anti-PD-1/PD-L1 pathway may be a potential therapeutic strategy for the treatment of TPE.

\section{Acknowledgements}

The present study was supported by grants from the National Natural Science Foundation of China (grant no. 81272610), the Science and Technology Support Program of Suzhou, China (grant no. SS201246), and the Science and Technology Development Program of Suzhou, China (grant no. SYS201467).

\section{References}

1. WHO global tuberculosis report 2015. www.who.int/iris/bitstre am/10665/191102/1/9789241565059_eng.pdf?ua=1.

2. Porcel JM: Tuberculous pleural effusion. Lung 187: 263-270, 2009.

3. Valdés L, Pose A, San José E and MartínezVázquez JM: Tuberculous pleural effusions. Eur J Intern Med 14: 77-88, 2003.

4. Khan FY, Hamza M, Omran AH, Saleh M, Lingawi M, Alnaqdy A, Rahman MO, Ahmedullah HS, Hamza A, Ani AA, et al: Diagnostic value of pleural fluid interferon-gamma and adenosine deaminase in patients with pleural tuberculosis in Qatar. Int J Gen Med 6: 13-18, 2013.

5. Azimi Q, Rezadoost B, Jalali Nadoushan M and Davati A: Evaluation of serum cyfra21-1 in patients with pleural effusion. Iran Red Crescent Med J 14: 613-616, 2012.

6. Dong H, Zhu G, Tamada K and Chen L: B7-H1, a third member of the B7 family, co-stimulates T-cell proliferation and interleukin-10 secretion. Nat Med 5: 1365-1369, 1999.

7. Liang SC, Greenwald RJ, Latchman YE, Rosas L, Satoskar A, Freeman GJ and Sharpe AH: PD-L1 and PD-L2 have distinct roles in regulating host immunity to cutaneous leishmaniasis. Eur J Immunol 36: 58-64, 2006. 
8. Ye ZJ, Zhou Q, Yin W, Yuan ML, Yang WB, Xiang F, Zhang JC, Xin JB, Xiong XZ and Shi HZ: Interleukin 22-producing CD4 ${ }^{+} \mathrm{T}$ cells in malignant pleural effusion. Cancer Lett 326: 23-32, 2012

9. Chen Y, Wang Q, Shi B, Xu P, Hu Z, Bai L and Zhang X: Development of a sandwich ELISA for evaluating soluble PD-L1 (CD274) in human sera of different ages as well as supernatants of PD-L1 $1^{+}$cell lines. Cytokine 56: 231-238, 2011.

10. Liu DM, Yan JC, Wang CP, Chen GH, Ding S, Liu PJ and Du RZ: The clinical implications of increased OX40 ligand expression in patients with acute coronary syndrome. Clin Chim Acta 397: 22-26, 2008.

11. Jiang J, Jiang J, Liu C, Zhang G, Gao L, Chen Y, Zhu R, Wang T, Wang F, Zhang X and Xue Q: Enhancement of membrane B7-H3 costimulatory molecule but reduction of its soluble form in multiple sclerosis. J Clin Immunol 33: 118-126, 2013.

12. Xing YF, Zhang ZL, Shi MH, Ma Y and Chen YJ: The level of soluble programmed death-1 in peripheral blood of patients with lung cancer and its clinical implications. Zhonghua Jie $\mathrm{He} \mathrm{He} \mathrm{Hu} \mathrm{Xi}$ Za Zhi 35: 102-106, 2012 (In Chinese).

13. Frigola X, Inman BA, Lohse CM, Krco CJ, Cheville JC, Thompson RH, Leibovich B, Blute ML, Dong H and Kwon ED: Identification of a soluble form of B7-H1 that retains immunosuppressive activity and is associated with aggressive renal cell carcinoma. Clin Cancer Res 17: 1915-1923, 2011

14. Muenst S, Soysal SD, Gao F, Obermann EC, Oertli D and Gillanders WE: The presence of programmed death 1(PD-1)-positive tumor-infiltrating lymphocytes is associated with poor prognosis in human breast cancer. Breast Cancer Res Treat 139: 667-676, 2013.

15. de Paus RA, van Wengen A, Schmidt I, Visser M, Verdegaal EM, van Dissel JT and van de Vosse E: Inhibition of the type I immune responses of human monocytes by IFN- $\alpha$ and IFN- $\beta$. Cytokine 61: $645-655,2013$.

16. Shi B, Du X, Wang Q, Chen Y and Zhang X: Increased PD-1 on CD4(+)CD28(-) T cell and soluble PD-1 ligand-1 in patients with T2DM: Association with atherosclerotic macrovascular diseases. Metabolism 62: 778-785, 2013.

17. Infante-Duarte $C$ and Kamradt $\mathrm{T}$ : Th1/Th2 balance in infection. Springer Semin Immunopathol 21: 317-338, 1999.

18. Yang WB, Liang QL, Ye ZJ, Niu CM, Ma WL, Xiong XZ, Du RH, Zhou Q, Zhang JC and Shi HZ: Cell origins and diagnostic accuracy of interleukin 27 in pleural effusions. PLoS One 7: e40450, 2012.

19. Light RW, Macgregor MI, Luchsinger PC and Ball WC Jr: Pleural effusions: The diagnostic separation of transudates and exudates. Ann Intern Med 77: 507-513, 1972.

20. Kastelik JA: Management of malignant pleural effusion. Lung 191: 165-175, 2013.

21. Tan EM, Cohen AS, Fries JF, Masi AT, McShane DJ, Rothfield NF, Schaller JG, Talal N and Winchester RJ: The 1982 revised criteria for the classification of systemic lupus erythematosus. Arthritis Rheum 25: 1271-1277, 1982.

22. Niederman MS, Mandell LA, Anzueto A, Bass JB, Broughton WA, Campbell GD, Dean N, File T, Fine MJ, Gross PA, et al: Guidelines for the management of adults with community-acquired pneumonia. Diagnosis, assessment of severity, antimicrobial therapy, and prevention. Am J Respir Crit Care Med 163: 1730-1754, 2001

23. Jurado JO, Alvarez IB, Pasquinelli V, Martínez GJ, Quiroga MF, Abbate E, Musella RM, Chuluyan HE and García VE: Programmed death (PD)-1: PD-ligand1/PD-ligand 2 pathway inhibits $\mathrm{T}$ cell effector functions during human tuberculosis. J Immunol 181: 116-125, 2008.

24. Reiley WW, Shafiani S, Wittmer ST, Tucker-Heard G, Moon JJ, Jenkins MK, Urdahl KB, Winslow GM and Woodland DL: Distinct functions of antigen-specific CD4 T cells during murine Mycobacterium tuberculosis infection. Proc Natl Acad Sci USA 107: 19408-19413, 2010.
25. Yin W, Tong ZH, Cui A, Zhang JC, Ye ZJ, Yuan ML, Zhou Q and Shi HZ: PD-1/PD-Ls pathways between CD4(+) T cells and pleural mesothelial cells in human uberculous pleurisy. Tuberculosis (Edinb) 94: 131-139, 2014

26. Porcel JM: Tuberculous pleural effusion. Lung 187: 263-270, 2009.

27. Xia H, Ye ZJ, Zhou Q, You WJ, Cui A, Wang XJ, Zhai K, Jin XG, Tong ZH and Shi HZ: IL-27 and IL-27-producing CD4+ T cells in human tuberculous pleural effusion. Tuberculosis (Edinb) 94: 579-588, 2014.

28. 28. Qama D, Choi WI, Kwon KY: Immune responses in the lungs of patients with tuberculous pleural effusion without pulmonary tuberculosis. BMC Immunol 13: 45, 2012.

29. Dong H, Strome SE, Salomao DR, Tamura H, Hirano F, Flies DB, Roche PC, Lu J, Zhu G, Tamada K, et al: Tumor-associated B7-H1 promotes T-cell apoptosis: A potential mechanism of immune evasion. Nat Med 8: 793-800, 2002.

30. Amarnath S, Costanzo CM, Mariotti J, Ullman JL, Telford WG, Kapoor V, Riley JL, Levine BL, June CH, Fong T, et al: Regulatory $\mathrm{T}$ cells and human myeloid dendritic cells promote tolerance via programmed death ligand-1. PLoS Biol 8: e1000302, 2010.

31. Trinath J, Maddur MS, Kaveri SV, Balaji KN and Bayry J: Mycobacterium tuberculosis promotes regulatory T-cell expansion via induction of programmed death-1 ligand 1 (PD-L1, CD274) on dendritic cells. J Infect Dis 205: 694-696, 2012.

32. Frigola $X$, Inman BA, Krco CJ, Liu X, Harrington SM, Bulur PA, Dietz AB, Dong H and Kwon ED: Soluble B7-H1: Differences in production between dendritic cells and T cells. Immunol Lett 142: 78-82, 2012

33. He XH, Xu LH and Liu Y: Identification of a novel splice variant of human PD-L1 mRNA encoding an isoform-lacking Igv-like domain. Acta Pharmacol Sin 26: 462-468, 2005.

34. Jiang J, Shi HZ, Liang QL, Qin SM and Qin XJ: Diagnostic value of interferon-gamma in tuberculous pleurisy: A metaanalysis. Chest 131: 1133-1141, 2007.

35. Lee SJ, Jang BC, Lee SW, Yang YI, Suh SI, Park YM, Oh S, Shin JG, Yao S, Chen L and Choi IH: Interferon regulatory factor- 1 is prerequisite to the constitutive expression and IFN-gamma-induced up-regulation of B7-H1 (CD274). FEBS Lett 580: 755-762, 2006.

36. Ma J, Yang B, Yu S, Zhang Y, Zhang X, Lao S, Chen X, Li B and Wu C: Tuberculosis antigen-induced expression of IFN- $\alpha$ in tuberculosis patients inhibits production of IL-1 $\beta$. FASEB J 28: 3238-3248, 2014.

37. Sutherland JS, Garba D, Fombah AE, Mendy-Gomez A, Mendy FS, Antonio M, Townend J, Ideh RC, Corrah T and Ota MO: Highly accurate diagnosis of pleural tuberculosis by immunological analysis of the pleural effusion. PLoS One 7: e30324, 2012.

38. Wang T, Lv M, Qian Q, Nie Y, Yu L and Hou Y: Increased frequencies of $T$ helper type 17 cells in tuberculous pleural effusion. Tuberculosis (Edinb) 91: 231-237, 2011.

39. Budak F, Uzaslan EK, Cangür S, Göral G and Oral HB Increased pleural soluble Fas Ligand (sFasL) levels in tuberculosis pleurisy and its relation with T-helper type 1 cytokines. Lung 186: 337-343, 2008.

40. Liu YC, Shin-Jung Lee S, Chen YS, Tu HZ, Chen BC and Huang TS: Differential diagnosis of tuberculous and malignant pleurisy using pleural fluid adenosine deaminase and interferon gamma in Taiwan. J Microbiol Immunol Infect 44: 88-94, 2011.

41. Ibrahim L, Salah M, Abd El Rahman A, Zeidan A and Ragb M: Crucial role of $\mathrm{CD} 4^{+} \mathrm{CD} 25^{+} \mathrm{FOXP} 3^{+} \mathrm{T}$ regulatory cell, interferon- $\gamma$ and interleukin-16 in malignant and tuberculous pleural effusions. Immunol Invest 42: 122-136, 2013. 